\title{
The Software Package PAOLAC: an embedment of the analytical code PAOLA within the CAOS problem-solving environment
}

\author{
Marcel Carbillet ${ }^{1, a}$, Laurent Jolissaint ${ }^{2}$, and Anne-Lise Maire ${ }^{1,3}$ \\ 1 UMR 6525 H. Fizeau - Université de Nice Sophia Antipolis (UNS) / Centre National de la Recherche \\ Scientifique (CNRS) / Observatoire de la Côte d'Azur (OCA), Parc Valrose, F-06108 Nice Cedex 2 \\ 2 Leiden Observatory, Niels Bohrweg 2, NL-2333 CA Leiden \\ 3 Master "Physique Fondamentale et Appliquée" $\Omega$ mega, UNS, Parc Valrose, F-06108 Nice Cedex 2
}

\begin{abstract}
We present the Software Package PAOLAC ("PAOLA within Caos") in its first distributed version. This new numerical simulation tool is an embedment of the analytical adaptive optics simulation code PAOLA ("Performance of Adaptive Optics for Large (or Little) Apertures") within the CAOS problem-solving environment. The main goal of this new tool is to allow an easier and direct comparison between studies performed with the analytical open-loop code PAOLA and studies performed with the end-to-end closed-loop Software Package CAOS ("Code for Adaptive Optics Systems"), with the final scope of better understanding how to take advantage from the two approaches: one analytical allowing extremely quick results on a wide range of cases and the other extremely detailed but with a computational and memory costs which can be impressive. The practical implementation of this embedment is briefly described, showing how this absolutely does not affect any aspect of the original code which is simply directly called from the CAOS global graphical interface through ad hoc modules. A comparison between end-to-end modelling and analytical modelling is hence also initiated, within the specific framework of wide-field adaptive optics at Dome C, Antarctica.
\end{abstract}

\section{The CAOS problem-solving environment}

The name "CAOS" was originally used to describe the Software Package CAOS [2] which permits end-to-end numerical modeling of adaptive optics (AO) systems. But it now also describes the CAOS problem-solving environment (PSE) [1], or "system", which allows to clearly separate in its own bosom the scientific part of the original software package from the global interface and structure of the tool and also the various routines of global utility, permitting to complete as well the whole suite with a number of other software packages covering a wider area of astronomical-optics-related scientifical topics. The CAOS PSE is so composed of:

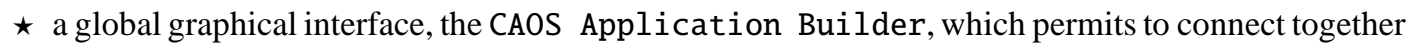
modules from the various software packages installed in order to define a simulation data flow,

$\star$ a library of utilities, the CAOS Library,

$\star$ and the software Packages, each of them being a collection of modules.

Concerning the various software Packages being developed, they actually cover a much larger domain than the original end-to-end $\mathrm{AO}$ one, going from generic astronomical image reconstruction to instrument-dedicated simulation tools. They can be very briefly described as follow:

$\star$ image reconstruction/deconvolution with the Software Package AIRY (Astronomical Image Reconstruction with Interferometry [5]),

$\star$ multiple deconvolution specialized for the instrument LINC-NIRVANA (Large binocular telescope INterferometric Camera - Near-InfraRed/Visible Adaptive iNterferometer for Astronomy, LN for short) with the Software Package AIRY-LN [6],

\footnotetext{
a e-mail: marcel.carbillet@unice.fr
} 


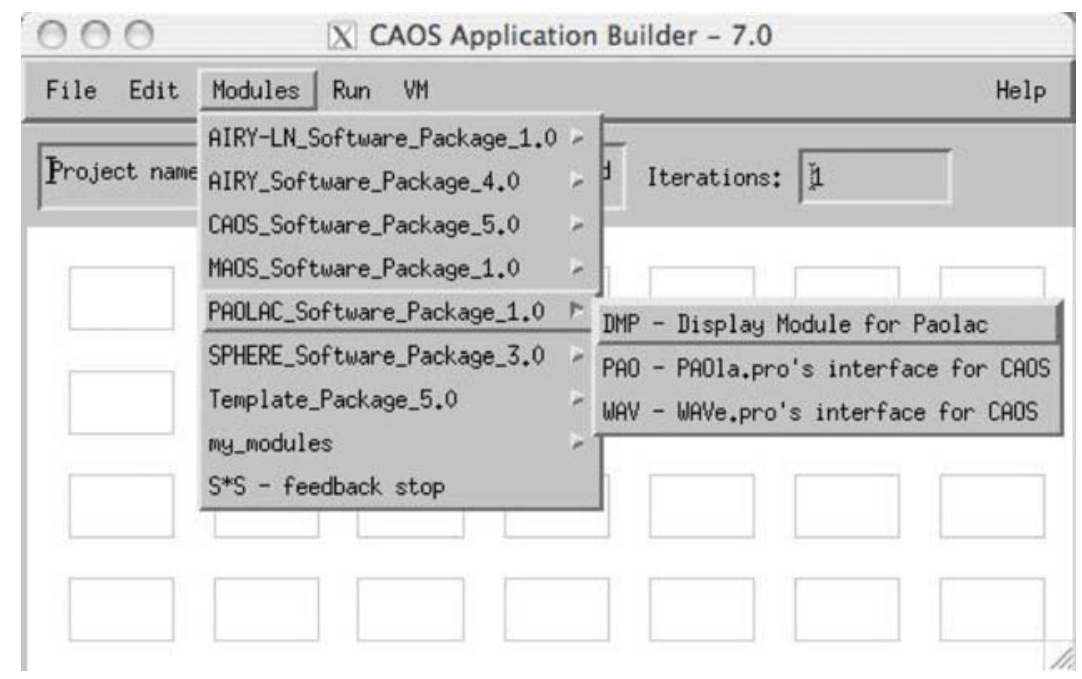

Fig. 1. The CAOS Application Builder, version 7.0, together with the list of installed software packages visible by pulling down the Modules menu. In particular, the few modules of the Software Package PAOLAC are shown.

$\star$ simulation of the data delivered by the Very Large Telescope instrument SPHERE (Spectro-Polarimetric High-contrast Exoplanet REsearch) with the Software Package SPHERE [3],

$\star$ and next multi-conjugate and ground-layer AO simulations with the Software Package MAOS (Multiple-reference AO Simulations).

On its side, the CAOS Library includes all the tools (routines or set of routines dedicated to a given task) that are, or can potentially be, used by the different software packages. For example, it includes since recently the oaa_lib library, a library of routines previously developed independently and which contains in particular a set of Zernike-specialized routines.

Within the CAOS Application Builder, from the pull-down menu Modules, the list of installed software packages is shown (see Fig. 1). From this list, and selecting PAOLAC, its modules can be selected and placed into the "worksheet" in order to compose a simulation project by combining together the modules, and defining the corresponding data flow with the only logical limitation due to inputs/outputs compatibility. In fact, a number of input/output data types are defined and for each of them a color is attributed within the CAOS Application Builder. As a consequence, a link traced between an output of the occurrence of a module and an input of another one must be between an output and an input corresponding to the same color, i.e. corresponding to the same data type.

After the simulation design step is completed, the IDL code implementing the simulation program is automatically generated and the whole structure of the simulation is saved as a project that can be restored for latter modifications and/or parameters upgrading. Note that parameters definition and upgrading are completely independent from the definition of the data flow, and hence the structure represented within the CAOS Application Builder of a simulation project: they do not affect in any way the structure itself of the simulation project. Hence the latter does not have to be saved again after parameters upgrading. This is part of the flexibility permitted by the modular structure of the CAOS PSE.

Note that by taking advantage from the "Virtual Machine" feature included in recent IDL versions, one can also now build an IDL-licence-free executable (thanks to the CAOS Application Builder button VM) made from any simulation project and which can be run afterwards on machines with neither the CAOS PSE nor the original PAOLA code installed, and, moreover, not even IDL. 
Table 1. Brief description of the present modules of the Software Package PAOLAC.

\begin{tabular}{l|l}
\hline Module & Purpose \\
\hline PAO - PAOla.pro's interface & - generate long-exposure PSF \& power spectrum \\
WAV - WAVe.pro's interface & - generate instantaneous PSF \& wavefront \\
DMP - Display Module for Paolac & - display inputs/outputs within PAOLAC \\
\hline
\end{tabular}

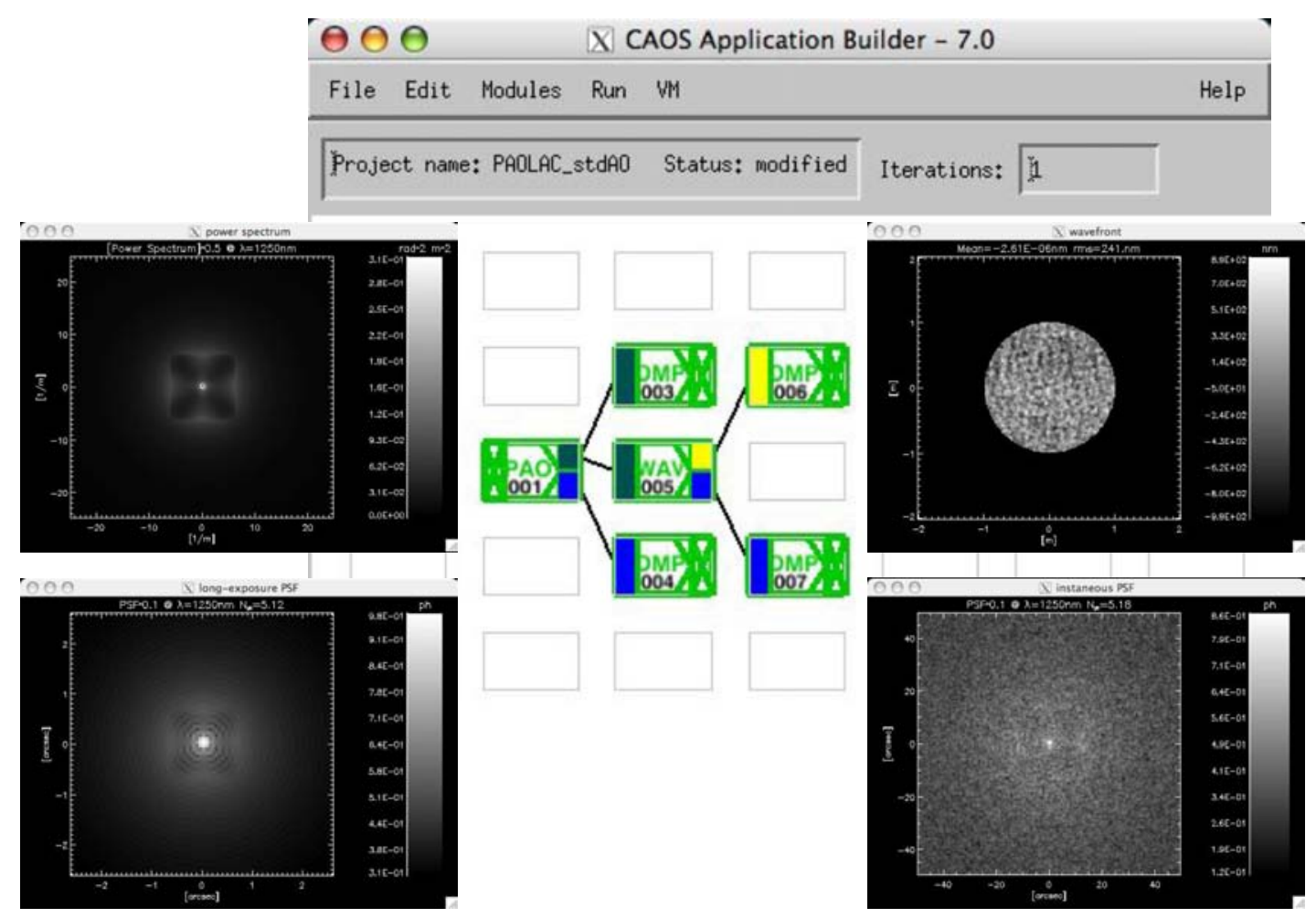

Fig. 2. A typical PAOLAC project (center), together with the displayed results: the long-exposure PSD (top left), the resulting long-exposure PSF (bottom left), the instantaneous post-AO wavefront (top right), and the corresponding instantaneous PSF (bottom right).

\section{The Software Package PAOLAC}

The Software Package PAOLAC is composed, at least for the present version 1.0, of two modules representative of the two main procedures of the original code PAOLA [7], namely paola.pro and wave.pro, together with one utility module made up for data display purposes, as briefly described inTab. 1. Figure 2 shows the way these modules can be simply used within the CAOS Application Builder in order to build a typical PAOLAC project.

Each term of the residual post-AO error budget is computed by module PAO, which is just an interface to the original PAOLA routine paola.pro. The basic concept under the analytical modeling performed starts from the modeling of the phase spatial power spectrum (PSD) to obtain finally the corresponding point-spread function (PSF), as represented by Fig. 3.

The PSF and PSD obtained are then delivered as the two outputs of module PAO. Instantaneous PSF and wavefronts are deduced from the previously-computed PSD, by module WAV, which is just an interface to the original PAOLA routine wave.pro, and which then delivers its two instantaneous outputs. Figure 4 shows the graphical user interface (GUI) of module PAO which permits to select the physical and numerical parameters relative to this module. The GUI relative to module WAV is much 


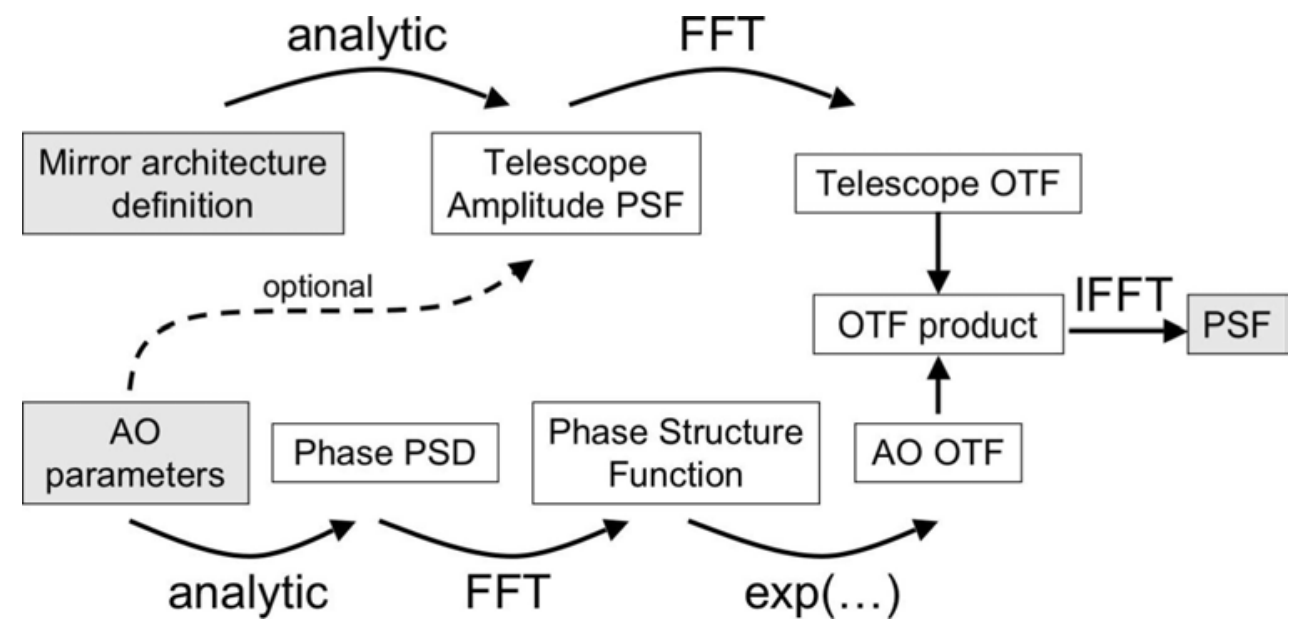

Fig. 3. Flow chart describing the modeling of the PSF within PAOLA (and hence also within PAOLAC). For more details see Jolissaint et al. [7] and Jolissaint et al. [8].

simpler since it only asks for the parameters file relative to the previously used occurrence of module PAO: in fact both of them roughly need the same set of physical and numerical parameters.

\section{An Example of Application: Wide-field AO at Dome C, Antarctica}

Following this work of embedment of the open-loop ${ }^{1}$ analytical code PAOLA within the CAOS PSE in order to build the new Software Package PAOLAC, we are presently comparing the results obtained with PAOLAC in one hand, and the end-to-end closed-loop Software Package CAOS in the other hand. As a preliminary test of this comparison study, which has to be deeply investigated in order to take advantage from the two approaches (one analytical allowing extremely quick results on a wide range of cases and the other extremely detailed but with a computational and memory costs which can be impressive), we here compare results of end-to-end simulations of two AO systems : a $10 \times 10$ subapertures Shack-Hartmann-based standard one, and a 3-guide-stars 15 arcmin-field-of-view groundlayer AO (GLAO) system, both embarked on a 2m-class near-infrared telescope for Dome C (called for now PLT - Pilot-Like Telescope), obtained and presented very recently [4], to the results obtained with PAOLAC by considering the same two AO systems. Figure 5 shows the state of the art of our on-going comparisons of the behavior of the standard AO system (left) and the 3-stars GLAO system (right), within the (large) field of view considered.

As mentioned before, these are very preliminary results, still suffering from some differences between the two modeling (e.g. the wavefront sensors CCD simulated are not exactly identical for the analytical code and the end-to-end code), but the global behavior in function of the angle in the field is reassuringly similar. This is enlightened when re-normalizing the value found with PAOLAC in the center of the field with the one found with the Software Package CAOS, as seen from Fig. 5 again.

\section{Availability of the code}

The Software Package PAOLAC is freely distributed such as any part of the CAOS PSE is, through the CAOS webpage http: //fizeau . unice.fr/caos. A dedicated mailing-list is also set up, in order to easily follow upgrades and the like of both the global interface/structure of the CAOS PSE and the

\footnotetext{
1 The last distributed version of PAOLA (6.3.2) is indeed still open-loop, but recent developments will lead to a closed-loop version available for distribution as soon as possible.
} 
M. Carbillet et al.: The Soft. Pack. PAOLAC: an embedment of PAOLA within the CAOS "system"

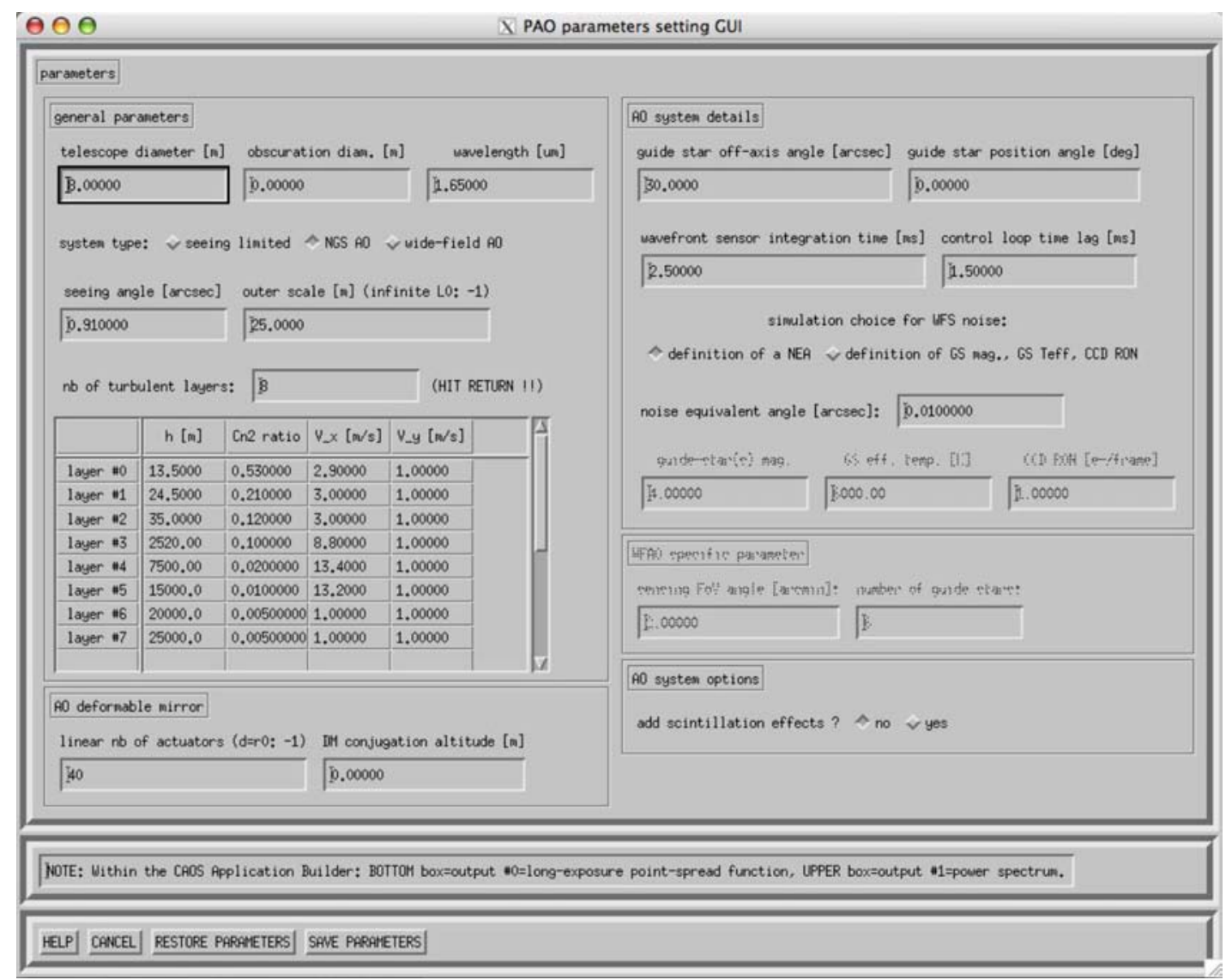

Fig. 4. GUI of module PAO which permits to select the physical and numerical parameters relative to this module.

Software Package PAOLAC itself. Subscription to the mailing-list is part of the installation instructions of the Software Package PAOLAC. Please note anyway that the original PAOLA code still needs to be obtained separately, by sending a request to jolissaint@strw.leidenuniv.nl.

\section{References}

1. M. Carbillet, C. Vérinaud, M. Guarracino, et al., in "Advancements in Adaptive Optics", D. Bonaccini, B. Ellerbroek, \& R. Ragazzoni Eds., SPIE Proc. 5490 (2) (2004), 550.

2. M. Carbillet, C. Vérinaud, B. Femenía, et al., Mon. Not. R. Astron. Soc. 356 (4) (2005), 1263.

3. M. Carbillet, A. Boccaletti, Ch. Thalmann, et al., in "Adaptive Optics Systems", N. Hubin, C. E. Max, \& P. L. Wizinowich, Eds., SPIE Proc. 7015 (2008), $70156 Z$.

4. M. Carbillet, A.-L. Maire, B. Le Roux, et al., in ARENA-3 Conf. Proc., EDPSciences (2009).

5. S. Correia, M. Carbillet, P. Boccacci, et al., Astron. Astrophys. 387 (2002), 733.

6. G. Desiderà, A. LaCamera, P. Boccacci, et al., in "Optical and Infrared Interferometry", M. Schöller, W. C. Danchi, \& F. Delplancke, Eds., SPIE Proc. 7013 (2008), 701340.

7. L. Jolissaint, J.-P. Véran, R. Conan, J. Opt. Soc. Am. A 23 (2) (2006a), 382.

8. L. Jolissaint, B. Ellerbroek, G. Angeli, in "Modeling, Systems Engineering, and Project Management for Astronomy II”, M. J. Cullum \& G. Z. Angeli, Eds., SPIE Proc. 6271 (2006b), 62711 C. 
First conference on Adaptive Optics for Extremely Large Telescopes
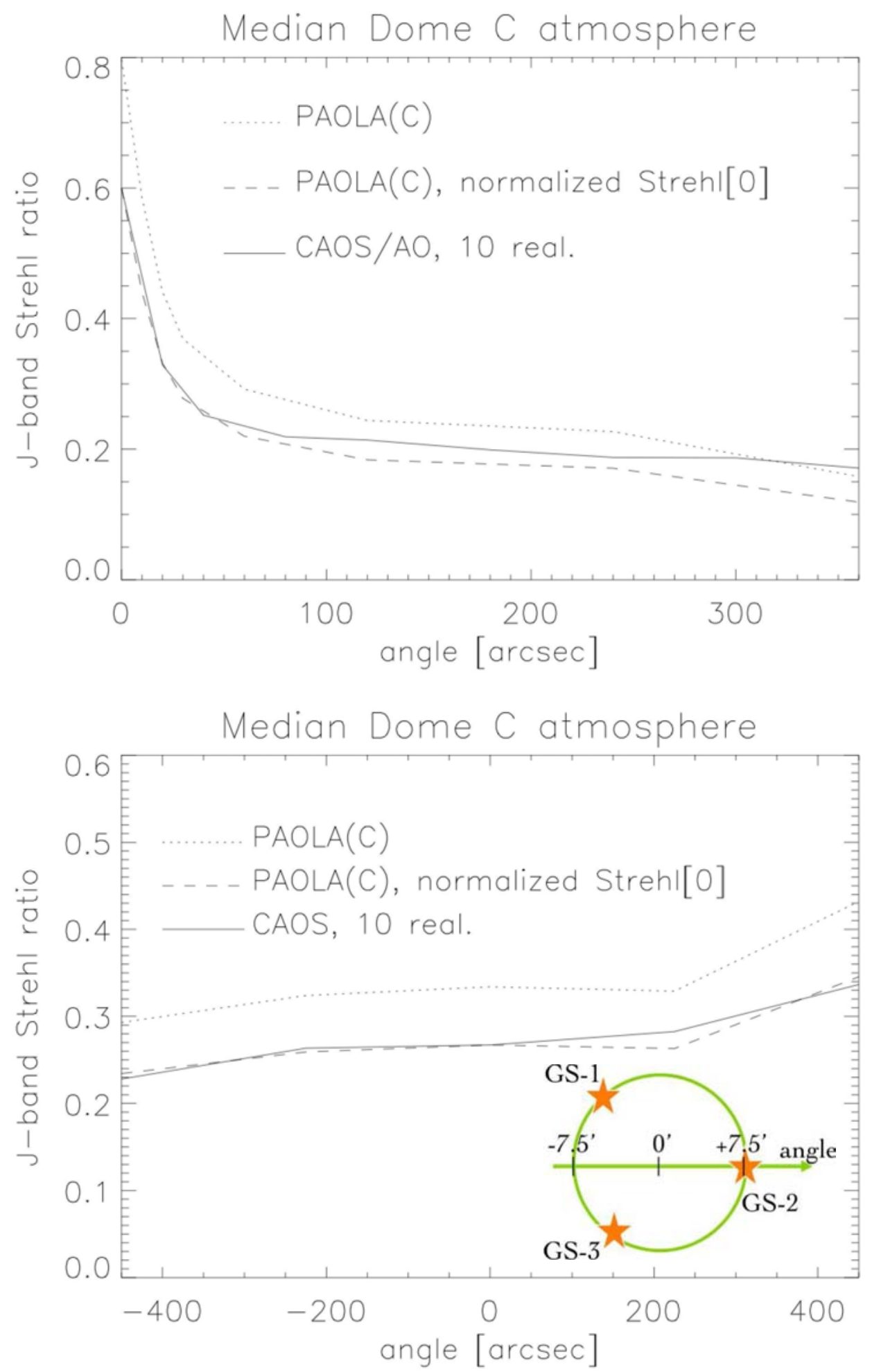

Fig. 5. State of the art of our on-going comparisons of the behavior of a standard AO system (left) and a 3-stars GLAO system (right), within the field of view considered. 